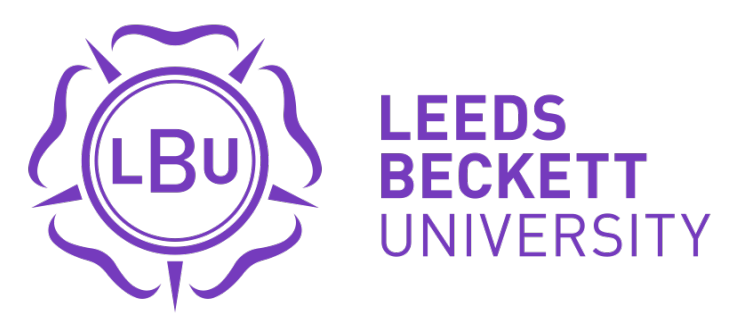

Citation:

Tsoularis, A (2014) Deterministic and stochastic optimal inventory control with logistic stockdependent demand rate. International Journal of Mathematics in Operational Research, 6 (1). 41 69. ISSN 1757-5850 DOI: https://doi.org/10.1504/IJMOR.2014.057851

Link to Leeds Beckett Repository record:

https://eprints.leedsbeckett.ac.uk/id/eprint/2259/

Document Version:

Article (Published Version)

The aim of the Leeds Beckett Repository is to provide open access to our research, as required by funder policies and permitted by publishers and copyright law.

The Leeds Beckett repository holds a wide range of publications, each of which has been checked for copyright and the relevant embargo period has been applied by the Research Services team.

We operate on a standard take-down policy. If you are the author or publisher of an output and you would like it removed from the repository, please contact us and we will investigate on a case-by-case basis.

Each thesis in the repository has been cleared where necessary by the author for third party copyright. If you would like a thesis to be removed from the repository or believe there is an issue with copyright, please contact us on openaccess@leedsbeckett.ac.uk and we will investigate on a case-by-case basis. 


\title{
Deterministic and stochastic optimal inventory control with logistic stock-dependent demand rate
}

\author{
A. Tsoularis \\ Business Rose Bowl, \\ Leeds Metropolitan University, \\ Portland Crescent, \\ Leeds LS1 3HB, UK \\ E-mail: a.tsoularis@leedsmet.ac.uk
}

\begin{abstract}
It has been suggested by many supply chain practitioners that in certain cases inventory can have a stimulating effect on the demand. In mathematical terms this amounts to the demand being a function of the inventory level alone. In this work we propose a logistic growth model for the inventory dependent demand rate and solve first the continuous time deterministic optimal control problem of maximising the present value of the total net profit over an infinite horizon. It is shown that under a strict condition there is a unique optimal stock level which the inventory planner should maintain in order to satisfy demand. The stochastic version of the optimal control problem is considered next. A bang-bang type of optimal control problem is formulated and the associated Hamilton-Jacobi-Bellman equation is solved. The inventory level that signifies a switch in the ordering strategy is worked out in the stochastic case.
\end{abstract}

Keywords: logistic growth; Euler-Lagrange equation; Legendre condition; Hamilton-Jacobi-Bellman equation; Wiener process; Itó conditions.

Reference to this paper should be made as follows: Tsoularis, A. (2014) 'Deterministic and stochastic optimal inventory control with logistic stock-dependent demand rate', Int. J. Mathematics in Operational Research, Vol. 6, No. 1, pp.41-69.

Biographical notes: A. Tsoularis obtained his BSc in Physics/Astrophysics (1982) and MSc in Operational Research (1984) from the University of London. He worked as an Engineer for the electricity industry in UK from 1989 to 1994 before obtaining his $\mathrm{PhD}$ in Cybernetics from Reading University in 1997. He moved to Massey University, New Zealand, in 1999 as a Senior Lecturer in Operational Research. In 2008, he returned to UK to join the Business School at Leeds Metropolitan University.

\section{Literature review}

High level of inventory can stimulate demand in some retail contexts (Balakrishan et al., 2004). Early evidence of this was presented in Wolfe (1968). The motivational effect was analysed from the marketing viewpoint in Levin at al. (1972) and subsequently by Baker and Urban (1988), Urban $(1992,1995)$ and Goh (1992). The idea is that large stock for certain products may generate higher demand. Baker and Urban (1988) were the first to 
propose a power function of the instantaneous inventory level for the demand rate, $r(x)=\alpha x^{\beta}$, where $r(x)$ is the demand rate, $x$ is the inventory level, $\alpha>0,0<\beta<1$. In the same vein Urban (1992) dealt with the determination of the optimal lot size ordered and delivered at the beginning of each order cycle in the context of economic order quantity $(E O Q)$ without the added restriction of zero ending-inventory. An analogous power form was suggested by Datta and Paul (2001) but with a variable parameter, $\alpha=\alpha(k)$, to reflect the mark-up rate in the price. Berman and Perry (2006) presented an EOQ-type model with piecewise constant and exponential demand rates and included holding cost functions, $h(x)=h_{0} x^{\gamma}$, for arbitrary $\gamma$. In a comprehensive overview of the literature of inventory-level dependent demand models, Urban (2005) classified them into Type I models and Type II models. In Type I models, the demand rate is a deterministic function of the initial stock level, whereas in Type II models, the demand rate is a function of the instantaneous inventory level. The vast majority of the references in Urban have focused on Type II models with instantaneous replacement (no backlogging) and profit considerations. Inventory deterioration was considered in a paper by Urban (1995) and another by Giri et al. (1996). None of these works incorporated the optimisation of an objective in inventory planning. Feichtinger and Hartl (1985) dealt with the determination of optimal pricing policy and production rate with non-linear price-dependent demand over a finite horizon by using optimal control theory. Khmelnitsky and Gerchak (2002) proposed a deterministic infinite horizon optimal control model incorporating price, inventory costs and production costs. Alfares (2007) considered the inventory policy for an item with a stock-level dependent demand rate and a storage-time dependent holding cost. The holding cost per unit of the item per unit time was assumed to be an increasing function of the time spent in storage. Alfares finally determined the optimal order quantity and the optimal cycle time. $\mathrm{Xu}$ (2009) studied a single-product, dynamic, nonstationary, stochastic inventory problem with capacity commitment, in which a buyer purchases a fixed capacity from a supplier at the beginning of a planning horizon and the buyer's total cumulative order quantity over the planning horizon is constrained with the capacity. The objective of the buyer is to choose the capacity at the beginning of the planning horizon and the order quantity in each period to minimise the expected total cost over the planning horizon. Xu identified conditions under which a myopic ordering policy is optimal and derived an equation for the optimal capacity commitment. He then used the optimal capacity and the myopic ordering policy to evaluate the effect of the various parameters on the minimum expected total cost over the planning horizon. Sarkar et al. (2011) dealt with an economic production quantity (EPQ) model for both continuous and discrete random demand of merchandise taking into account items of imperfect quality reworked at a cost and maximised the associated expected integrated profit by standard analytical calculus method. Xu et al. (2011) studied an inventory system in which a supplier supplies a non-stationary Poisson demand using two mutually substitutable products over a selling season of $T$ periods, with a single replenishment opportunity at the beginning of the season. Adopting a stochastic dynamic programming formulation, $\mathrm{Xu}$ et al. first proved the concavity of the value function, thereby facilitating the solution of the optimal replenishment quantities. Chen et al. (2012) considered a setting in which inventory plays both promotional and service roles, that is, higher inventories not only improve service levels but also stimulate demand by serving as a promotional tool. Specifically their study was the periodic-review inventory systems in which the demand in each period is uncertain but increases with the inventory level. 


\section{The demand rate function}

In this article we introduce an inventory-level-dependent function for the demand rate that is analogous to the logistic model for population growth used in population ecology (Tsoularis and Wallace, 2002). The logistic growth model has the form

$$
\frac{d x}{d t}=\alpha x\left(1-\frac{x}{D}\right)
$$

where $x$ is the current biological population and $D$ is the carrying capacity which reflects the available environmental resources for sustaining the population. The growth rate is proportional to the difference between the available resources and the necessary resources for current sustenance. The factor $\alpha$, is the intrinsic per capita growth rate.

In the context of modelling inventory dependent demand we put forward the idea that if the actual demand is a continuous function of time, $y(t)$, its rate of growth, $\frac{d y}{d t}$, will evolve according to the current inventory, $x(t)$, thus:

$$
\frac{d y}{d t}=\alpha x\left(1-\frac{x}{D}\right)
$$

Here $D$ is the stock level where demand ceases to grow, reaching either a saturation point or is halted due to storage limitations. This imposes a limit on the growth in demand which can, for instance, be attributed to a saturating effect. As $D$ is an arbitrary parameter, it can be large or small depending on the nature of the product being offered which is assumed to be replenished at a rate, $u$. The function, $\alpha x\left(1-\frac{x}{D}\right)$, is concave and positive for all $x$ (actual demand, $y(t)$, is always increasing), and will be increasing $\left(\dot{y}_{x}>0\right)$ for $x<\frac{D}{2}$ and decreasing $\left(\dot{y}_{x}<0\right)$ for $x>\frac{D}{2}$, always at a decreasing rate $\left(\dot{y}_{x x}<0\right)$. The maximum growth rate, $\frac{\alpha D^{2}}{4}$, occurs at $x=\frac{D}{2}$. So for $x<\frac{D}{2}$, the demand rate behaves like a Type II model, as 2 classified by Urban (2005). The essential feature of (1) is that it captures in sequence growth, saturation and decline in sales as inventory levels become increasingly abundant. Aoki and Yoshikawa (2002) highlight the utility of logistic growth in various industrial sectors. Similar modes of product demand are described in Nahmias (1997).

\section{Problem formulation}

In this section we formulate the problem of maximising discounted net profit from the sale of products driven by demand obeying (1). We ignore setup costs and item deterioration and assume no backlogging and zero lead times. The objective is to maximise the present value of the net revenue (profit less inventory costs and order costs) over an infinite horizon. 
We adopt the following notation:

$x(t)$

is the inventory level at time $t$

$x_{0} \in(0, D)$ is the initial inventory at time $t=0$

$\alpha x\left(1-\frac{x}{D}\right)$ is the inventory dependent demand rate

$u(t) \quad$ is the order (production) rate per unit time up to a maximum rate $U$

$p \quad$ is the fixed revenue per unit item

$h \quad$ is the fixed inventory cost per unit item

$c \quad$ is the fixed order (production) cost per unit item $(c<p)$

$r \quad$ is the fixed discount rate

$\alpha \quad$ is the fixed coefficient regulating the demand rate per unit inventory item per unit time

$D \quad$ is the maximum stock level possible or demand saturation inducing inventory

The problem is to maximise the present value of the total net profit:

$$
J\left(x_{0}\right)=\int_{0}^{\infty} e^{-r t}\left(p \alpha x\left(1-\frac{x}{D}\right)-h x-c u\right) d t
$$

subject to the following state equation and constraints:

$$
\begin{aligned}
& \dot{x}(t)=u(t)-\alpha x\left(1-\frac{x}{D}\right), \\
& x(0)=x_{0}, \\
& 0 \leq u(t) \leq U .
\end{aligned}
$$

Starting from an initial inventory, $x(0)=x_{0}$, an optimal production schedule, $0 \leq u^{*}(t) \leq U$, must be found for which the functional (2) attains its maximum value. The presence of the discount term, $e^{-r t}$, in the integrand, and the boundedness of $p \alpha x\left(1-\frac{x}{D}\right)-h x-c u$ ensures convergence for the integral in (2).

\section{Derivation of the optimal control policy}

Applying standard procedure, as for instance in Kamien and Schwartz (2001), the Hamilton-Jacobi-Bellman (H-J-B) linear first order differential equation is obtained: 


$$
r V^{*}(x)=\max _{u(t)}\left(\left(p \alpha x\left(1-\frac{x}{D}\right)-h x-c u\right)+\left(u+\alpha x\left(1-\frac{x}{D}\right)\right) \frac{d V^{*}}{d x}\right)
$$

where $V^{*}(x)$ is the optimal net current value profit when the current inventory is $x(t)$. It follows from (4) that the optimal control policy depends on the algebraic sign of $\frac{d V^{*}}{d x}-c$ :

$$
u^{*}(t)=\left\{\begin{array}{c}
U \text { if } \frac{d V^{*}}{d x}>c \\
0 \text { if } \frac{d V^{*}}{d x}<c \\
0 \leq u \leq U \text { if } \frac{d V^{*}}{d x}=c
\end{array}\right.
$$

As long as the marginal inventory value, $\frac{d V^{*}}{d x}$, exceeds the order cost, $c$, the optimal policy is to order at the maximum rate, $U$, otherwise no orders are placed. When $\frac{d V^{*}}{d x}=c$, the choice of $u$ is formally undetermined; any order policy in the range $[0, U]$ does not affect the solution of (4). There are then two subsets of the inventory state space, one in which $\frac{d V^{*}}{d x}>c$, and another in which $\frac{d V^{*}}{d x}<c$, that are separated by a particular inventory value, $x^{*}$, which is an accumulation point of the two subsets and at which the control may assume any arbitrary value. Once the inventory level exceeds $x^{*}$, its marginal value drops below the order cost, $c$, and orders must cease until the inventory falls below $x^{*}$ and its marginal value begins to rise again by resuming orders. In order to establish the existence or otherwise of such threshold inventory level we turn to the calculus of variations. Since our problem is one-dimensional infinite horizon autonomous problem linear in $u(t)$, we can eliminate $u(t)$ from (3) and substitute the resulting expression in (2). Substitution for $u(t)=\dot{x}+\alpha x\left(1-\frac{x}{D}\right)$ in (2) yields:

$$
\begin{aligned}
J & =\int_{0}^{\infty} e^{-r t} L(x, \dot{x}) d t \\
& =\int_{0}^{\infty} e^{-r t}(M(x)+N(x) \dot{x}) d t
\end{aligned}
$$

subject to the constraints

$$
\begin{aligned}
& x(0)=x_{0}, \\
& -\alpha x\left(1-\frac{x}{D}\right) \leq \dot{x} \leq U-\alpha x\left(1-\frac{x}{D}\right),
\end{aligned}
$$


where

$$
\begin{aligned}
& L(x, \dot{x})=M(x)+N(x) \dot{x} \\
& M(x)=(p-c) \alpha x\left(1-\frac{x}{D}\right)-h x, \\
& N(x)=-c .
\end{aligned}
$$

Application of the Euler-Lagrange equation, $\frac{d}{d t}\left(e^{-r t} \frac{\partial L}{\partial \dot{x}}\right)=e^{-r t} \frac{\partial L}{\partial \dot{x}}$, gives

$$
\alpha(p-c)\left(1-\frac{2 x}{D}\right)-h-r c=0
$$

Equation (9) is an algebraic equation in a single variable, $x$, and has a unique stationary solution, $x^{*}$, given by

$$
x^{*}=\frac{D}{2}\left(1-\frac{r c+h}{\alpha(p-c)}\right)
$$

Since $p>c, x^{*}<\frac{D}{2}$. The condition

$$
\alpha>\frac{r c+h}{p-c}
$$

must be observed for $x^{*}$ to exist. (11) states that for an optimum inventory level to exist the intrinsic demand growth rate, $\alpha$, must exceed the ratio, $\frac{r c+h}{p-c}$, that involves all the monetary parameters of the problem and represents the fraction of the net income from selling a single item, $p-c$, that is absorbed by the accumulated interest on the order cost, $r c$, and the holding cost, $h$.

The Euler-Lagrange equation as well as the Legendre condition, $e^{-r t} \frac{\partial^{2} L}{\partial \dot{x}^{2}}=0$, provide necessary but not sufficient conditions for optimality. The sufficiency condition is furnished by proving the concavity of the integrand, $e^{-r t} L(x, \dot{x})$. Since both conditions

$$
\begin{aligned}
& e^{-r t} \frac{\partial^{2} L}{\partial x^{2}}=e^{-r t} \frac{\partial^{2} M}{\partial x^{2}}=-2 e^{-r t} \frac{\alpha}{D}(p-c)<0, \\
& e^{-r t}\left(\frac{\partial^{2} L}{\partial x^{2}} \frac{\partial^{2} L}{\partial \dot{x}^{2}}-\left(\frac{\partial^{2} L}{\partial x \partial \dot{x}}\right)^{2}\right)=0,
\end{aligned}
$$

hold, the concavity of the integrand is proved. 
The optimal solution to the optimisation problem defined by (2) and (3) is to move from $x_{0}$ to $x^{*}$ as quickly as possible and then remain at $x^{*}$ thereafter. This is the so-called most rapid approach path $(M R A P)$ approach. Spence and Starrett (1975) provide a detailed analysis of this approach in the autonomous case whereas Hartl and Feichtinger (1987) derive the Euler equation in the non-autonomous case ( $M$ and $N$ are functions of time, $t$, as well as state, $x$ ). We offer a brief graphical proof of the optimality of $M R A P$ below.

Figure 1 shows two alternative paths from $x_{0}$ to $x^{*}$.

Figure 1 Alternative paths from $x_{0}$ to $x^{*}$

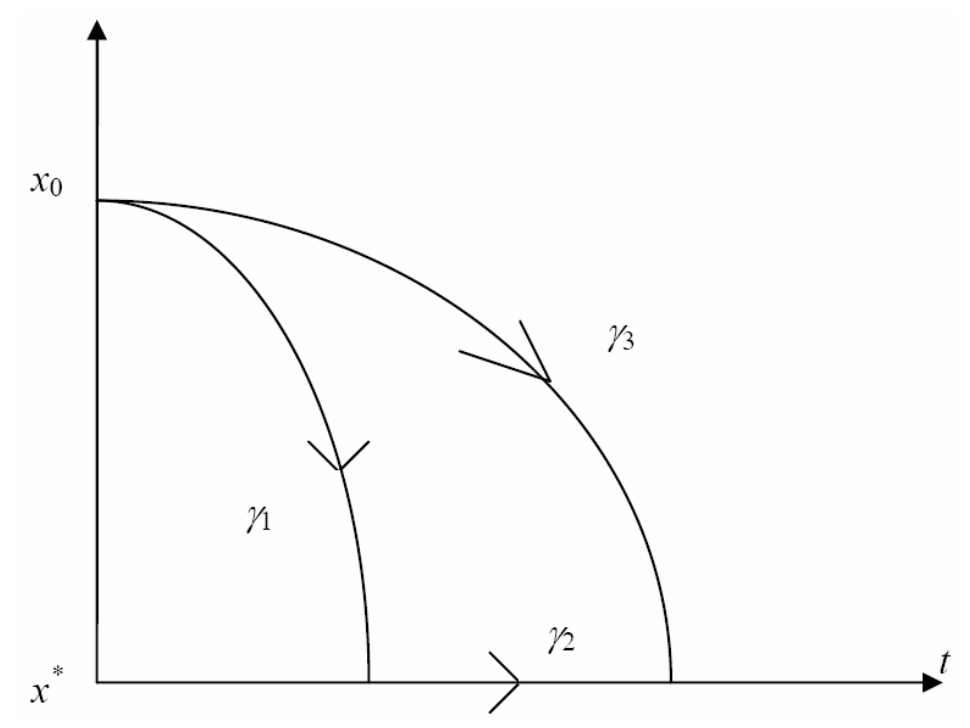

We note first that $\int_{0}^{\infty} e^{-r t}(M(x)+N(x) \dot{x}) d t=\int_{0}^{\infty} e^{-r t}(M(x) d t+N(x) d x)$, which is a line integral. Assume that the path starts from the initial state, $x_{0}$, and ends at $x^{*}<x_{0}$. We evaluate this line integral along two paths, $\gamma_{1} \gamma_{2}$ and $\gamma_{3}$, that is, $\int_{\gamma_{1} \gamma_{2}} e^{-r t}(M(x) d t+N(x) d x)$ and $\int_{\gamma_{3}} e^{-r t}(M(x) d t+N(x) d x)$. Then we form the difference between these two line integrals:

$$
\begin{aligned}
& \int_{\gamma_{1} \gamma_{2}} e^{-r t}(M(x) d t+N(x) d x)-\int_{\gamma_{3}} e^{-r t}(M(x) d t+N(x) d x) \\
& =\int_{\gamma_{1} \gamma_{2} \gamma_{3}} e^{-r t}(M(x) d t+N(x) d x)
\end{aligned}
$$


This is a line integral around the closed curve $\gamma_{1} \gamma_{2} \gamma_{3}$ in the counterclockwise (positive) sense, which, by application of Green's Theorem, can be written as a double integral:

$$
\begin{aligned}
& \int_{\gamma / 12 \gamma_{3}} e^{-r t}(M(x) d t+N(x) d x)=\iint\left(\frac{\partial\left(e^{-r t} N(x)\right)}{\partial t}-\frac{\partial\left(e^{-r t} M(x)\right)}{\partial x}\right) d t d x \\
& =-\iint e^{-r t}\left(r N(x)+\frac{d M}{d x}\right) d t d x
\end{aligned}
$$

The double integral expresses the difference between the values of two functionals along two different paths. The path $\gamma_{1} \gamma_{2}$ is the path that approaches $x^{*}$ most rapidly and will be the optimal one if the integrand is negative, that is if,

$$
r N(x)+\frac{d M}{d x}<0
$$

A similar argument applies when $x_{0}<x^{*}$, provided that the reverse condition

$$
r N(x)+\frac{d M}{d x}>0
$$

now holds.

In our problem, $\frac{d M}{d x}+r N(x)=\alpha(p-c)\left(1-\frac{2 x}{D}\right)-h-r c$ is positive if $x_{0}<x^{*}$, negative if $x_{0}>x^{*}$, and 0 if $x_{0}=x^{*}$. It is therefore optimal to get to $x^{*}$ from $x_{0}$ at the maximum rate. If $x_{0}>x^{*}$, the maximum rate can be realised by not placing any orders $(u=0)$ so that the inventory will decline at the rate, $-\alpha x\left(1-\frac{x}{D}\right)$. If $x_{0}<x^{*}$, the maximum rate can be realised by placing orders at the maximum rate $(u=U)$ so that the inventory will rise at the rate, $U-\alpha x\left(1-\frac{x}{D}\right)$. In short, If $x_{0}=x^{*}, x^{*}$ must be maintained by placing orders at exactly the same rate the inventory changes, that is, $u^{*}=\alpha x^{*}\left(1-\frac{x^{*}}{D}\right)$.

In accordance to the MRAP we have:

$$
x_{0}=\left\{\begin{array}{c}
>x^{*}, u=0 \\
=x^{*}, u^{*}=\alpha x^{*}\left(1-\frac{x^{*}}{D}\right) \\
<x^{*}, u=U
\end{array}\right.
$$

The optimal policies outlined by (5) and (12) are of course equivalent. Implementation of (5) requires solving directly for the value function and (12) for the state equation. 


\section{Analytical solutions to the state equation (3)}

In this section, we derive general analytical solutions for the inventory variable for the two extreme values, 0 and $U$, of the control variable, $u(t)$. However, the various solutions for $u=U$ are only relevant for any initial inventory value, $x_{0}<x^{*}$. Next we solve the differential equation (3) with $u(t)=0$ and $u(t)=U$. Before we do so we are going to need the roots of the quadratic equation, $u-\alpha x\left(1-\frac{x}{D}\right)=0$ :

$$
\begin{gathered}
x_{1}=\frac{D+\sqrt{D^{2}-\frac{4 u D}{\alpha}}}{2} \\
x_{2}=\frac{D-\sqrt{D^{2}-\frac{4 u D}{\alpha}}}{2}
\end{gathered}
$$

\subsection{Solution for $U<\frac{\alpha D}{4}$}

In this case the roots $x_{1}$ and $x_{2}$ are both real. The differential equation has the following solution:

$$
x(t)=\frac{x_{1}-\frac{x_{2}\left(x_{0}-x_{1}\right)}{x_{0}-x_{2}} \exp \left(t \sqrt{\alpha^{2}-\frac{4 u \alpha}{D}}\right)}{1-\frac{x_{0}-x_{1}}{x_{0}-x_{2}} \exp \left(t \sqrt{\alpha^{2}-\frac{4 u \alpha}{D}}\right)}
$$

Since $\left(\frac{d f}{d x}\right)_{x=x_{1}}>0$ and $\left(\frac{d f}{d x}\right)_{x=x_{2}}<0, x_{1}$ is an unstable equilibrium and $x_{2}$ is an asymptotically stable equilibrium. The solution (14) is well defined for all $x_{0} \in\left(0, x_{2}\right) \cup\left(x_{2}, D\right)$. At $x_{0}=x_{2}$, the inventory remains at this level for ever. For any other initial inventory value the inventory will eventually settle towards $x_{2}$. The presence of the stable equilibrium, $x_{2}$, places certain restrictions on the location of the initial inventory value, $x_{0}$. We examine all possible cases in detail and to that effect we calculate the time, $t^{*}$, needed to reach $x^{*}$ from (14):

$$
t^{*}=\frac{1}{\sqrt{\alpha^{2}-\frac{4 u \alpha}{D}}} \ln \left(\frac{\left(x^{*}-x_{1}\right)\left(x_{0}-x_{2}\right)}{\left(x^{*}-x_{2}\right)\left(x_{0}-x_{1}\right)}\right)
$$


$1 x_{2}<x_{0}<x^{*}$ : the inventory can build up to $x^{*}$ level at time $t^{*}$, where the optimal steady state order rate, $u^{*}=\alpha x^{*}\left(1-\frac{x^{*}}{D}\right)$, can be exercised thereafter. Since $x_{2}$ and $U$ are functionally dependent, $\mathrm{U}$ is subject to the following constraint:

$$
U<\frac{\alpha D}{4}-\frac{D}{4 \alpha}\left(\frac{r c+h}{p-c}\right)^{2}
$$

$2 x_{0}<x_{2}<x^{*}$ : the inventory will be attracted asymptotically towards $x_{2}$ before it gets to $x^{*}$ and will remain at this level thereafter. In this case $x^{*}$ is unattainable, as it can also be seen from the non-existence of a real value for $t^{*}$ in (15).

$3 x_{0}<x^{*} \leq x_{2}$ : the inventory can eventually build up to the desired value, $x^{*}$, at or before $x_{2}$. The reverse condition to (16) holds in this case

$$
\frac{\alpha D}{4}-\frac{D}{4 \alpha}\left(\frac{r c+h}{p-c}\right)^{2} \leq U<\frac{\alpha D}{4}
$$

\subsection{Solution for $U<\frac{\alpha D}{4}$}

When $U<\frac{\alpha D}{4}, x_{1}=x_{2}=\frac{D}{2}$, and the solution is:

$$
x(t)=\frac{D}{2}+\frac{D\left(2 x_{0}-D\right)}{\alpha\left(D-2 x_{0}\right) t+2 D}
$$

The solution (18) is well defined in the time interval $[0, \infty)$ for all $x_{0} \in\left(\frac{D}{2}\right]$. For $x_{0} \in\left(\frac{D}{2}, D\right)$ the solution is defined on the time interval, $t \in\left[0, \frac{2 D}{\alpha\left(2 x_{0}-D\right)}\right)$. The inventory level, $x_{0}=\frac{D}{2}$, is an unstable equilibrium.

For an initial inventory value, $x_{0} \leq x^{*}<\frac{D}{2}$, the time taken to reach $x^{*}$ is given by

$$
t^{*}=\frac{2(p-c)}{r c+h}+\frac{2 D}{\alpha\left(2 x_{0}-D\right)}
$$




\subsection{Solution for $U<\frac{\alpha D}{4}$}

The roots are complex conjugates with positive real part, hence unstable. The solution to the differential equation in this case is:

$$
\begin{aligned}
& x(t)=\frac{D}{2}+\frac{D \omega}{\alpha} \tan (\phi+\omega t), \\
& \tan \phi=\frac{x_{0}-\frac{D}{2}}{\frac{D \omega}{\alpha}}, \quad \omega=\sqrt{\frac{U \alpha}{D}-\frac{\alpha^{2}}{4}}
\end{aligned}
$$

The solution (20) is periodic with period, $\frac{\pi}{\omega}$, and is well defined for all $x_{0} \in(0, D)$ in the entire time interval, $t \in[0, \infty)$, except at the discrete time instants,

$$
t_{n}=\frac{(2 n+1) \frac{\pi}{2}-\phi}{\omega}, n=0,1,2, \ldots
$$

For $x_{0}<x^{*}<\frac{D}{2}$, the monotonicity of the tangent function guarantees that $x^{*}$ will be reached at time, $t^{*}$, given by

$$
t^{*}=\frac{1}{\omega}\left(\arctan \left(\frac{r c+h}{2 \omega(c-p)}\right)-\phi\right)
$$

\subsection{Solution for $u=0$}

Finally, when $u=0$, the solution is:

$$
x(t)=\frac{D x_{0} e^{-\alpha t}}{x_{0} e^{-\alpha t}+D-x_{0}}
$$

In this case the inventory will asymptotically drop to the asymptotically stable value, $x=0$, as $t \rightarrow \infty$, from any initial value, $x_{0} \in(0, D)$. From an initial inventory value, $x_{0}>x^{*}$, the time taken to reach, $x^{*}$, is

$$
t^{*}=\frac{1}{\alpha} \ln \left(\frac{x_{0}(\alpha(p-c)+r c+h)}{(\alpha(p-c)-r c-h)\left(D-x_{0}\right)}\right)
$$




\section{Derivation of the optimal net profit}

In Section 4, we stated the H-J-B equation:

$$
r V^{*}(x)=\max _{u(t)}\left(\left(p \alpha x\left(1-\frac{x}{D}\right)-h x-c u\right)+\left(u-\alpha x\left(1-\frac{x}{D}\right)\right) \frac{d V^{*}}{d x}\right)
$$

This is a first order ordinary linear differential equation which is normally solved by solving for the maximising $u^{*}$ in terms of $x$ and $\frac{d V^{*}}{d x}$ first, and subsequently substituting the result into the equation to obtain a modified differential equation that must be solved with an appropriate boundary condition. Since $u$ is a linear term however, an analytical expression for $u$ is not obtainable. As we have already established that $u$ can assume its two extreme values, 0 and $U$, two differential equations must be solved instead:

$$
\begin{aligned}
& r V_{1}^{*}(x)=\max _{u(t)}\left(\left(p \alpha x\left(1-\frac{x}{D}\right)-h x-c U\right)+\left(U-\alpha x\left(1-\frac{x}{D}\right)\right) \frac{d V_{1}^{*}}{d x}\right) \text { for } x<x^{*} \\
& r V_{2}^{*}(x)=\max _{u(t)}\left(\left(p \alpha x\left(1-\frac{x}{D}\right)-h x\right)+\alpha x\left(1-\frac{x}{D}\right) \frac{d V_{2}^{*}}{d x}\right) \text { for } x<x^{*}
\end{aligned}
$$

To ensure the continuity of $V\left(x^{*}\right)$, it is sufficient that the condition, $V_{1}^{*}\left(x^{*}\right)=V_{2}^{*}\left(x^{*}\right)=V^{*}\left(x^{*}\right)$, is satisfied, as well as the condition for switching the optimal policy, $\left(\frac{d V^{*}}{d x}\right)_{x=x^{*}}=c \cdot V^{*}\left(x^{*}\right)$ is the current net optimal profit value when the current inventory is $x^{*}$, given by

$$
V^{*}\left(x^{*}\right)=\frac{(p-c) \alpha x^{*}\left(1-\frac{x^{*}}{D}\right)-h x^{*}}{r}
$$

From (26) it is straightforward to verify that $\left(\frac{d V^{*}}{d x}\right)_{x=x^{*}}=c$. The present value of the value function (26) is $e^{-r t^{*}} V^{*}\left(x^{*}\right)$, where $t^{*}$ is the time the stock level, $x^{*}$, is reached.

The solutions to (24) and (25) are

$$
\begin{aligned}
& V_{1}^{*}(x)=\frac{1}{g_{1}(x)}\left(\int g_{1}(x)\left(c U+h x-p \alpha x\left(1-\frac{x}{D}\right)\right) d x+k_{1}\right) \\
& V_{2}^{*}(x)=\frac{1}{g_{2}(x)}\left(\int g_{2}(x)\left(h x-p \alpha x\left(1-\frac{x}{D}\right)\right) d x+k_{2}\right)
\end{aligned}
$$


where $k_{1}, k_{2}$ are integration constants to be determined from the conditions $\left(\frac{d V_{1}^{*}}{d x}\right)_{x=x^{*}}=\left(\frac{d V_{2}^{*}}{d x}\right)_{x=x^{*}}=c$ and $V_{1}^{*}\left(x^{*}\right)=V_{2}^{*}\left(x^{*}\right)=V^{*}\left(x^{*}\right)$, the boundary, $x^{*}$, and

$$
\begin{aligned}
& g_{1}(x)=\exp \left(-\int \frac{r}{U-\alpha x\left(1-\frac{x}{D}\right)} d x\right) \\
& g_{2}(x)=\exp \left(\int \frac{r}{\alpha x\left(1-\frac{x}{D}\right)} d x\right)
\end{aligned}
$$

are the integrating factors worked out explicitly in Section 5.

\section{The stochastic inventory model demand growth function}

We now allow the demand to possess a certain degree of randomness and assume that after a small time interval, $d t$, the replenished inventory will on average have changed by the anticipated amount, $\left(u-\alpha x\left(1-\frac{x}{D}\right)\right) d t$, with variance, $\sigma^{2} x^{2} d t$, we can write down a stochastic differential equation for the inventory dynamics

$$
d x=\left(u-\alpha x\left(1-\frac{x}{D}\right)\right) d t+\sigma x d w
$$

The parameter, $\sigma$, is the diffusion parameter and $d w$ is the Wiener process, a normal random variable, $N(0, d t)$. We have written (31) in a differential form as the derivative of $d w$ is usually interpreted as white noise which cannot be formally integrated. A unique solution to (31) exists if both Itó conditions hold (Fleming and Rishel, 1975). The first is the linear growth condition

$$
\begin{aligned}
& \left|u-\alpha x\left(1-\frac{x}{D}\right)\right| \leq K(1+|x|) \\
& |\sigma x| \leq K(1+|x|)
\end{aligned}
$$

and the other is the Lipschitz condition

$$
\begin{aligned}
& \left|-\alpha x_{1}\left(1-\frac{x_{1}}{D}\right)+\alpha x_{2}\left(1-\frac{x_{2}}{D}\right)\right| \leq L\left|x_{1}-x_{2}\right| \\
& \left|\sigma\left(x_{1}-x_{2}\right)\right| \leq L\left|x_{1}-x_{2}\right|
\end{aligned}
$$


for some independent constants, $K$ and $L$. Both conditions are met for bounded $\alpha, \sigma$ and $u$. The solution to $(31)$ is the stochastic integral equation

$$
x(t)=x_{0}+\int_{0}^{t}\left(u-\alpha x\left(1-\frac{x}{D}\right)\right) d t+\int_{0}^{t} \sigma x d w, x_{0}=x(0) \ll D, w(0)=0
$$

Figure 2 shows ten sample paths and the mean inventory for $x_{0}=10, u=10, \alpha=0.2$, $\sigma=0.3, D=400$

Figure 2 Simulated solution of stochastic integral equation (32) (see online version for colours)

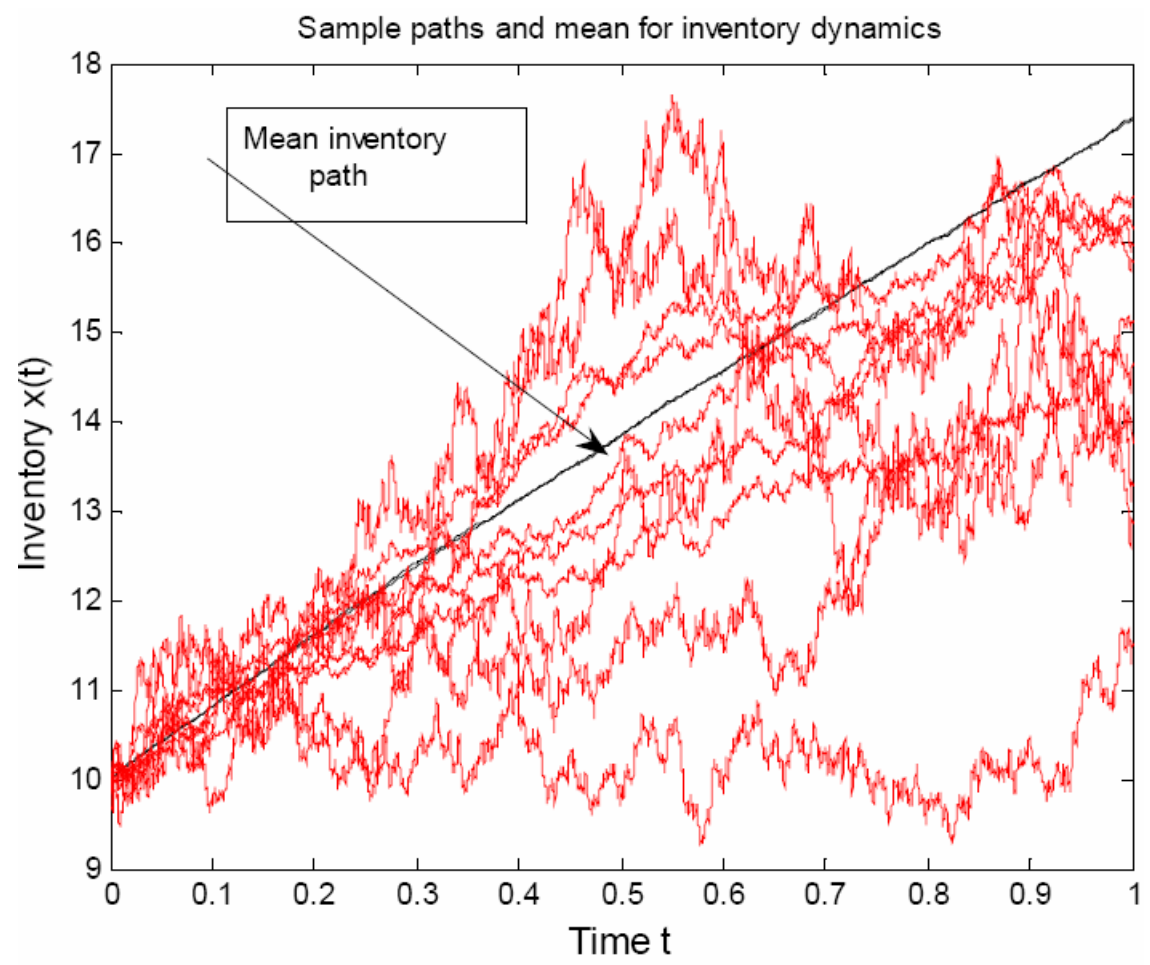

\section{The stochastic optimal control problem}

This paper looks next at the problem of discounted profit maximisation over an infinite horizon by selling a product for which inventory evolves according to (31). There have been a few important articles on optimal production involving white noise processes. Sethi and Thompson (1981) considered a convex production-inventory model which determines production rates over time to minimise an integral representing a discounted quadratic loss function with white noise in the dynamics of the inventory process. Bensoussan et al. (1984) considered an infinite horizon stochastic production planning 
problem with a discounted quadratic function designed to minimise the squared deviation from a desired inventory and production level. Fleming et al. (1987) considered an infinite horizon stochastic production planning problem with demand assumed to be a continuous-time Markov chain. Sethi et al. (1992) considered an infinite horizon stochastic production planning problem with capacity and demand assumed to be finite state Markov chains and established the existence of a threshold inventory level, or turnpike level, such that production takes place when below or at this level and no production above it. Dohi et al. (1995) dealt with an optimal production planning problem with the same objective as Bensoussan et al. (1984) but with demand assumed to be a mean-reverting Markovian diffusion process. A paper that is close to the spirit of the present work is the work of Berling and Martinez-de-Albéniz (2011). These authors considered the problem of exogenous stochastic demand following a Poisson distribution and a variable replenishment price evolving as a geometric Brownian motion or Ornstein-Uhlenbeck process and focused on determining the optimal stock level as a function of a series of threshold prices. It is worth noting that none of the aforementioned papers allow for inventory-dependent demand, which is what we present in this paper.

We consider the objective of maximising the expected net profit from selling a product with fixed price, $p$. Holding costs are incurred continuously at constant rate, $h$, per item and the fixed ordering cost per item is $c$. There is no backlogging and the lead times are zero. The retailer calculates the gross return, $p \alpha x\left(1-\frac{x}{D}\right)$, based on the expected demand. The net undiscounted profit is then, $\operatorname{pax}\left(1-\frac{x}{D}\right)-h x-c u$. Our objective is to maximise the expected value of the net discounted profit with discount rate, $r$, over an infinite horizon:

$$
V\left(x_{0}\right)=\max _{u(t)} E\left[\int_{0}^{\infty} e^{-r t}\left(p \alpha x\left(1-\frac{x}{D}\right)-h x-c u\right) d t \mid x(0)=x_{0}\right]
$$

subject to the stochastic differential equation (32), shown again below:

$$
d x=\left(u-\alpha x\left(1-\frac{x}{D}\right)\right) d t+\sigma x d w, x(0)=x_{0}, \ll D, w(0)=0
$$

and the control constraint:

$$
0 \leq u \leq U
$$

The second order derivative of the concave profit function is continuous and is therefore bounded by the polynomial growth condition, $\left|p \alpha x\left(1-\frac{x}{D}\right)-h x-x u\right| \leq K(1+x+u)$, for some constant $K$ (Fleming and Rishel, 1975). We assume that $p>\frac{h}{\alpha}$, that is, the price is larger in magnitude than the holding cost to demand growth parameter ratio so that holding inventory is cost effective if the growth in demand is sufficiently high. 
The H-J-B equation (Malliaris and Brock, 1982) for the stochastic control problem is a differential equation for the current value, $V(x)$ :

$$
r V=\max _{u(t)}\left(\left(p \alpha x\left(1-\frac{x}{D}\right)-h x-c u\right)+\left(u-\alpha x\left(1-\frac{x}{D}\right)\right) \frac{d V}{d x}+\frac{1}{2} \sigma^{2} x^{2} \frac{d^{2} V}{d x^{2}}\right)
$$

The second order linear differential equation (36) is linear in $u$. The optimal control decision, $u^{*}$, will then depend on the sign of $\frac{d V}{d x}-c$ :

$$
u^{*}=\left\{\begin{array}{c}
0 \text { if } \frac{d V}{d x}<c \\
u \in[0, U] \text { if } \frac{d V}{d x}=c \\
U \text { if } \frac{d V}{d x}>c
\end{array}\right.
$$

The optimal control law (37) suggests that the set of inventory states is divided into two distinct regions separated by a boundary on which the marginal profit, $\frac{d V}{d x}$, equals the unit order cost, $c$, and where $x$ attains a specific value, $x^{*}$. The problem then is to determine the stock level, $x^{*}$, at which $\left(\frac{d V}{d x}\right)_{x=x^{*}}=c$. If the stock happens to exceed the specific value, $x^{*}$, the optimal policy is to place no orders $\left(u^{*}=0\right)$ until the stock reaches $x^{*}$, whereas if the stock level has fallen below $x^{*}$, the optimal ordering policy is to order the maximum possible quantity $\left(u^{*}=U\right)$ until the stock reaches $x^{*}$. Once the stock level hits $x^{*}$, any admissible ordering policy, $u^{*} \in[0, U]$ is acceptable since $\left(\frac{d V}{d x}\right)_{x=x^{*}}=c$. Due to the random disturbance present in the system, the inventory will momentarily move away from $x^{*}$, but the extreme order policy, $u^{*}=0$ or $u^{*}=U$ will kick in to restore the inventory level back to $x^{*}$. Since the choice of control does not affect the solution of (36), the optimal control, $u^{*}=\alpha x^{*}\left(1-\frac{x^{*}}{D}\right)$, could be used to eliminate the drift in the stochastic differential equation (34). Excursions from $x^{*}$ would then be drift-free geometric Brownian motions of the form, $x(t)=x^{*} \exp \left(-\frac{\sigma^{2} t}{2}+\sigma w(t)\right)$ with expected value $[x(t)]=x^{*}$ and zero variance.

\section{The solution to the H-J-B equation when $D \rightarrow \infty$}

In this section we solve the optimal control problem when $D$ is for practical purposes infinite so the demand growth is linear, as the solution will aid us in getting the solution 
for finite $D$. When the demand saturation level, $D$, is infinite, the term, $\frac{\alpha x^{2}}{D}$, drops out from both the profit function and the diffusion process. The inventory dynamics is now

$$
d x=(u-\alpha x) d t+\sigma x d w, x(0)=x_{0}, w(0)=0
$$

and the profit functional is

$$
V^{*}\left(x_{0}\right)=\max _{u(t)} E\left[\int_{0}^{\infty} e^{-r t}(p \alpha x-h x-c u) d t \mid x(0)=x_{0}\right]
$$

The H-J-B second order differential equation is in this case given by

$$
\frac{1}{2} \sigma^{2} x^{2} \frac{d^{2} V^{*}}{d x^{2}}+(u-\alpha x) \frac{d V^{*}}{d x}-r V_{1}^{*}=(h-p \alpha) x+c u
$$

We produce two separate solutions to the H-J-B equation, one with solution, $V_{1}^{*}(x)$, when $u^{*}=U$ and $\frac{d V^{*}}{d x}-c>0$, and another with solution, $V_{2}^{*}(x)$, when $u^{*}=0$ and $\frac{d V^{*}}{d x}-c<0$. Continuity and differentiability of the solution at the threshold value, $x^{*}$, requires that the boundary conditions

$$
\begin{aligned}
& V_{1}^{*}\left(x^{*}\right)=V_{2}^{*}\left(x^{*}\right) \\
& \left(\frac{d V_{1}^{*}}{d x}\right)_{x=x^{*}}=\left(\frac{d V_{2}^{*}}{d x}\right)_{x=x^{*}}=c \\
& \left(\frac{d^{2} V_{1}^{*}}{d x^{2}}\right)_{x=x^{*}}=\left(\frac{d^{2} V_{2}^{*}}{d x^{2}}\right)_{x=x^{*}}
\end{aligned}
$$

be met. These conditions will be exploited to provide analytical forms for the current value function at the threshold inventory level, $x^{*}$.

\subsection{The H-J-B equation for $u^{*}=U$}

The H-J-B equation is:

$$
\frac{1}{2} \sigma^{2} x^{2} \frac{d^{2} V_{1}^{*}}{d x^{2}}+(u-\alpha x) \frac{d V_{1}^{*}}{d x}-r V_{1}^{*}=(h-p \alpha) x+c U
$$

$x=0$ is an irregular singular point of the second order differential equation but every other point, $x \neq 0$, is an ordinary point. We can therefore use a Taylor series expansion, $V_{1}^{*}(x)=\sum_{n=0}^{\infty} a_{n}(x-\xi)^{n}$, for the solution around any admissible state, $\xi \neq 0$. We transform powers of the variable, $x$, and rewrite the coefficients as polynomials of powers of $(x-\xi)$ thus: 


$$
\begin{aligned}
& x^{2}=(x-\xi)^{2}+2 \xi(x-\xi)+\xi^{2}, \\
& U-\alpha x=(U-\alpha \xi)-\alpha(x-\xi), \\
& (h-p \alpha) x+c U=(h-p \alpha)(x-\xi)+c U+\xi(h-p \alpha)
\end{aligned}
$$

Inserting the Taylor series into the differential equation we obtain:

$$
\begin{aligned}
& \sum_{n=0}^{\infty}\left(\frac{\sigma^{2} n(n-1)}{2} a_{n}+\sigma^{2} \xi n(n+1) a_{n+1}+\frac{\sigma^{2} \xi^{2}}{2}(n+1)(n+2) a_{n+2}\right)(x-\xi)^{n} \\
& +\sum_{n=0}^{\infty}\left((U-\alpha \xi)(n+1) a_{n+1}-n \alpha a_{n}-r a_{n}\right)(x-\xi)^{n} \\
& =(h-p \alpha)(x-\xi)+c U+\xi(h-p \alpha)
\end{aligned}
$$

Setting coefficients on both sides equal to 0 , the first two inhomogeneous terms are:

$$
\begin{aligned}
& n=0: \sigma^{2} \xi^{2} a_{2}+(U-\alpha \xi) a_{1}-r a_{0}=c U+\xi(h-p \alpha) \\
& n=1: \sigma^{2} \xi a_{2}+3 \sigma^{2} \xi^{2} a_{3}+2(U-\alpha \xi) a_{2}-\alpha a_{1}-r a_{1}=h-p \alpha
\end{aligned}
$$

The two initial coefficients, $a_{0}$ and $a_{1}$, can in principle, be determined by the appropriate boundary conditions. From them we obtain the third and fourth coefficients, $a_{2}$ and $a_{3}$ :

$$
\begin{aligned}
& a_{2}=\frac{c U+\xi(h-p \alpha)}{\sigma^{2} \xi^{2}}+\frac{t}{\sigma^{2} \xi^{2}} a_{0}-\frac{(U-\alpha \xi)}{\sigma^{2} \xi^{2}} a_{1} \\
& a_{3}=\frac{h-p \alpha}{3 \sigma^{2} \xi^{2}}+\frac{(\alpha+r)}{3 \sigma^{2} \xi^{2}} a_{1}-\frac{2\left(U-\alpha \xi+\sigma^{2} \xi\right)}{2 \sigma^{2} \xi^{2}} a_{2}
\end{aligned}
$$

The remaining coefficients, $a_{n}$, are calculated from the following recursive relationship:

$$
a_{n+2}=\frac{\left(2 n \alpha+2 r-n(n-1) \sigma^{2}\right) a_{n}-2(n+1)\left(n \sigma^{2} \xi+U-\alpha \xi\right) a_{n+1}}{(n+1)(n+2) \sigma^{2} \xi^{2}}, n \geq 2
$$

\subsection{The H-J-B equation for $u^{*}=0$}

In what follows the generic point $\xi$ is replaced by $x^{*}$. For $u^{*}=0$ the $\mathrm{H}-\mathrm{J}-\mathrm{B}$ equation is the inhomogeneous second order Euler equation:

$$
\left.\frac{1}{2} \sigma^{2} x^{2} \frac{d^{2} V_{2}^{*}}{d x^{2}}-\alpha x\right) \frac{d V_{2}^{*}}{d x}-r V_{2}^{*}=(h-p \alpha) x
$$

This can be solved, by the standard method of variation of parameters to give:

$$
V_{2}^{*}(x)=\frac{(h-p \alpha) x^{3}}{\left(3-\rho_{1}\right)\left(3-\rho_{2}\right)}+k_{1} x^{\rho_{1}}+k_{2} x^{\rho_{2}}
$$


where $k_{1}, k_{2}$ are integration constants to be determined from the appropriate boundary conditions, and the exponents, $\rho_{1}$ and $\rho_{2}$ are given by

$$
\begin{gathered}
\rho_{1}=\frac{\sigma^{2}+2 \alpha+\sqrt{\left(\sigma^{2}+2 \alpha\right)^{2}+8 r \sigma^{2}}}{2 \sigma^{2}}>1, \\
\rho_{2}=\frac{\sigma^{2}+2 \alpha+\sqrt{\left(\sigma^{2}+2 \alpha\right)^{2}+8 r \sigma^{2}}}{2 \sigma^{2}}<0
\end{gathered}
$$

At $x=0$, we impose the natural boundary condition, $V_{2}^{*}(0)=0$, and consequently the term, $k_{2}$, associated with the negative root, $\rho_{2}$, must be forced to equal zero. We present two different solutions for $V_{2}^{*}(x)$ :

$1 \quad \rho_{1} \neq 3$ :

$$
V_{2}^{*}(x)=\frac{(h-p \alpha) x^{3}}{\left(3-\rho_{1}\right)\left(3-\rho_{2}\right)}+k_{1} x^{\rho_{1}}
$$

There is only one unknown parameter, $k_{1}$, to be determined in (49). To that effect we use the condition, $\frac{d V_{2}^{*}}{d x}=c$. We have then

$$
\left(\frac{d V_{2}^{*}}{d x}\right)_{x=x^{*}}=c=\frac{3(h-p \alpha)\left(x^{*}\right)^{2}}{\left(3-\rho_{1}\right)\left(3-\rho_{2}\right)}+\rho_{1} k_{1}\left(x^{*}\right)^{\rho_{1}-1}
$$

whence,

$$
k_{1}=\frac{c}{\rho_{1}}\left(x^{*}\right)^{1-\rho_{1}}-\frac{3(h-p \alpha)\left(x^{*}\right)^{2}}{\rho_{1}\left(3-\rho_{1}\right)\left(3-\rho_{2}\right)}\left(x^{*}\right)^{3-\rho_{1}}
$$

Inserting (51) into (49) we obtain the final form of the value function

$$
V_{2}^{*}(x)=\frac{(h-p \alpha) x^{3}}{\left(3-\rho_{1}\right)\left(3-\rho_{2}\right)}+\left(\frac{x}{x^{*}}\right)^{\rho_{1}}\left(\frac{c x^{*}}{\rho_{1}}+\frac{3(p \alpha-h) x^{3}}{\rho_{1}\left(3-\rho_{1}\right)\left(3-\rho_{2}\right)}\left(x^{*}\right)^{3}\right)
$$

Finally, we need to determine the threshold value, $x^{*}$. We have

$$
\left(\frac{d^{2} V_{2}^{*}}{d x^{2}}\right)_{x=x^{*}}=a_{2}=\frac{6(h-p \alpha) x^{*}}{\left(3-\rho_{1}\right)\left(3-\rho_{2}\right)}+\rho_{1}\left(\rho_{1}-1\right) k_{1}\left(x^{*}\right)^{\rho_{1}-2}
$$

However, by virtue of (53), $a_{2}$ is also given by

$$
a_{2}=\frac{(h-p \alpha+\alpha c)}{\sigma^{2} x^{*}}+\frac{r}{\sigma^{2}\left(x^{*}\right)^{2}} V_{2}^{*}\left(x^{*}\right)
$$


Equating (53) to (54) and solving the quadratic equation in $x^{*}$ we have

$$
x^{*}=\sqrt{\frac{\left(3-\rho_{2}\right)\left(c \sigma^{2} \rho_{1}^{2}+\left(p \alpha-h-\alpha c-c \sigma^{2}\right) \rho_{1}-r c\right)}{\left(3 \sigma^{2} \rho_{1}+r\right)(p \alpha-h)}}
$$

$x^{*}$ exists if $c \sigma^{2} \rho_{1}^{2}+\left(p \alpha-h-\alpha c-c \sigma^{2}\right) \rho_{1}-r c>0$. We prove that this is indeed so next. Since $(p \alpha-h) \rho_{1}>0$, it is sufficient to prove that the inequality, $c \sigma^{2} \rho_{1}^{2}-\left(\alpha c+c \sigma^{2}\right) \rho_{1}-r c>0$, is also always valid. There are two roots to the quadratic equation, $c \sigma^{2} \rho_{1}^{2}-\left(\alpha c+c \sigma^{2}\right) \rho_{1}-r c=0$, which are given by $\frac{\sigma^{2}+\alpha \pm \sqrt{\left(\sigma^{2}+\alpha\right)^{2}+4 r \sigma^{2}}}{2 \sigma^{2}}$. Since by definition, $\rho_{1}>0$, only the positive root is relevant for the proof, namely $\frac{\sigma^{2}+\alpha+\sqrt{\left(\sigma^{2}+\alpha\right)^{2}+4 r \sigma^{2}}}{2 \sigma^{2}}<\rho_{1}$. It immediately follows then that the inequality, $c \sigma^{2} \rho_{1}^{2}-\left(\alpha c+c \sigma^{2}\right) \rho_{1}-r c>0$, always holds. The existence of $x^{*}$ is therefore conclusively proved.

$2 \rho_{1}=3$ :

In this case, $\sigma^{2}=\alpha+\frac{r}{3}$ and $\rho^{2}=-\frac{2 r}{3 \alpha+r}$, and there is a different solution to the inhomogeneous Euler equation given by

$V_{2}^{*}(x)=x^{3}\left(\frac{p \alpha-h}{\left(3-\rho_{2}\right)^{2}}+k_{1}-\frac{p \alpha-h}{3-\rho_{2}} \ln x\right)$

where the term, $k_{2} x^{\rho_{2}}$, has been dropped again for the same reason as for the case $\rho_{1} \neq 3$. The term, $x_{3} \ln x \rightarrow 0$ as $x \rightarrow 0$, according to L'Hospital's rule, and presents no problem. We have again

$\left(\frac{d V_{2}^{*}}{d x}\right)_{x=x^{*}}=c=\left(x^{*}\right)^{2}\left(\frac{\rho_{2}(p \alpha-h)}{\left(3-\rho_{2}\right)^{2}}+3 k_{1}\right)-\frac{3(p \alpha-h)}{3-\rho_{2}}\left(x^{*}\right)^{2} \ln x^{*}$

whence,

$k_{1}=\frac{c}{3\left(x^{*}\right)^{2}}+\frac{(p \alpha-h)}{3-\rho_{2}}\left(\ln x^{*}-\frac{\rho_{2}}{3\left(3-\rho_{2}\right)}\right)$

We have then the current value function

$V_{2}^{*}(x)=x^{3}\left(\frac{c}{3\left(x^{*}\right)^{2}}+\frac{p \alpha-h}{3-\rho_{2}}\left(\frac{1}{3}-\ln \left(\frac{x}{x^{*}}\right)\right)\right)$ 
Finally, solving $\left(\frac{d^{2} V_{2}^{*}}{d x^{2}}\right)_{x=x^{*}}=\frac{\alpha(c-p)+h}{\sigma^{2} x^{*}}+\frac{r V_{2}^{*}\left(x^{*}\right)}{\sigma^{2}\left(x^{*}\right)^{2}}$, for $x^{*}$, we obtain

$$
x^{*}=\sqrt{\frac{(c r+3 \alpha p+3 \alpha c-3 h)(9 \alpha+5 r)}{(3 \alpha+r)(9 \alpha+4 r)(p \alpha-h)}}
$$

The threshold inventory level, $x^{*}$, varies inversely with the diffusion parameter, $\sigma$, exhibiting a hyperbolic relationship of the form, $x^{*} \sim \frac{1}{\sigma}$. This indicates that large random perturbations in the interactive inventory-demand system call for a switch in the optimal ordering strategy when the stock falls considerably. Also higher ordering costs, $c$, lead to higher threshold inventory levels. Figure 3 displays an instance of this relationship for $r=0.03, p=2.5, \alpha=0.2, h=0.1, c=0.25$, for $\sigma \in[0,1]$.

Figure 3 Threshold inventory-diffusion inter-dependence (see online version for colours)

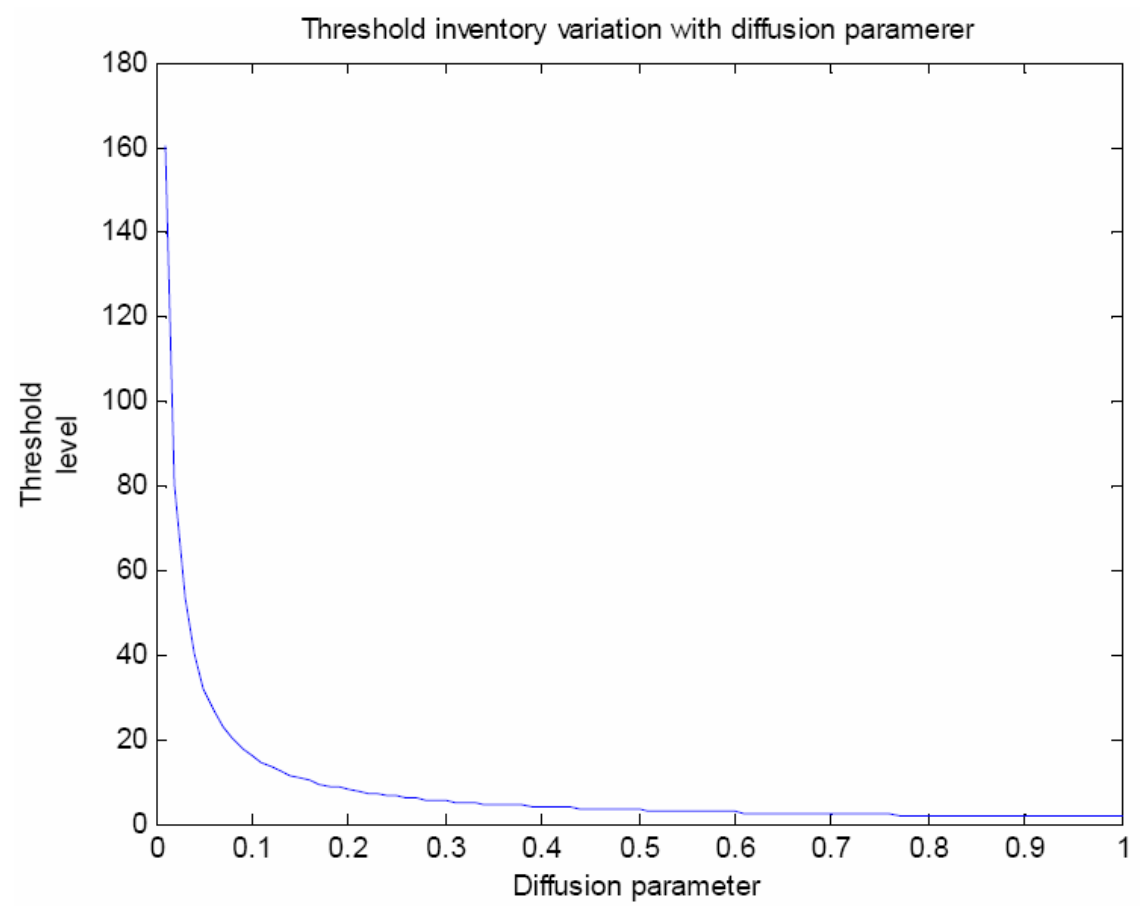

10 The solution to the H-J-B equation when $D<\infty$

Again in this section we solve the H-J-B equation twice, first for $u^{*}=U$, and then for $u^{*}=0$. 


\subsection{Solution to the $H-J-B$ equation for $u^{*}=U$}

The equation when $u^{*}=U$ is:

$$
\frac{1}{2} \sigma^{2} x^{2} \frac{d^{2} V_{1}^{*}}{d x^{2}}+\left(U-\alpha x\left(1-\frac{x}{D}\right)\right) \frac{d V_{1}^{*}}{d x}-r V_{1}^{*}=h x-p \alpha x\left(1-\frac{x}{D}\right)+c U
$$

$x=0$ is an irregular singular point of the second order differential equation (61), but every other point, $x \neq 0$, is an ordinary point. We can therefore use a Taylor series expansion, $V_{1}^{*}(x)=\sum_{n=0}^{\infty} a_{n}(x-\xi)^{n}$, for the solution around any non-zero inventory state, $\xi \neq 0$. We transform powers of the variable, $x$, and rewrite the coefficients as Taylor series expansions thus:

$$
\begin{aligned}
& x^{2}=(x-\xi)^{2}+2 \xi(x-\xi)+\xi^{2} \\
& U-\alpha x\left(1-\frac{x}{D}\right) \\
& =\frac{\alpha}{D}(x-\xi)^{2}+\alpha\left(\frac{2 \xi}{D}-1\right)(x-\xi)+U-\alpha \xi\left(1-\frac{\xi}{D}\right), h x-p \alpha x\left(1-\frac{x}{D}\right)+c U \\
& =\frac{p \alpha}{D}(x-\xi)^{2}+\left(p \alpha\left(\frac{2 \xi}{D}-1\right)+h\right)(x-\xi)+h \xi-p \alpha \xi\left(1-\frac{\xi}{D}\right)+c U
\end{aligned}
$$

Inserting the transformed coefficients into (61) and equating the coefficients of like powers on both sides we obtain for $n=0$ :

$$
a_{2}=\frac{r}{\sigma^{2}\left(x^{*}\right)^{2}} a_{0}+\frac{\alpha(c-p)\left(1-\frac{x^{*}}{D}\right)+h}{\sigma^{2} x^{*}}
$$

10.2 Solution to the H-J-B equation for $u^{*}=0$

For $u^{*}=0$ the equation becomes

$$
\frac{1}{2} \sigma^{2} x^{2} \frac{d^{2} V_{2}^{*}}{d x^{2}}-\alpha x\left(1-\frac{x}{D}\right) \frac{d V_{2}^{*}}{d x}-r V_{2}^{*}=h x-p \alpha x\left(1-\frac{x}{D}\right)
$$

The solution to the homogeneous version of (63)

$$
\frac{1}{2} \sigma^{2} x^{2} \frac{d^{2} V_{2}^{*}}{d x^{2}}-\alpha x\left(1-\frac{x}{D}\right) \frac{d V_{2}^{*}}{d x}-r V_{2}^{*}=0
$$


is found by substitution of the Frobenius series, $V_{2}^{*}(x)=\sum_{n=0}^{\infty} a_{n} x^{\beta+n}$, into (64), which yields the indicial equation:

$$
a_{0}\left(\sigma^{2} \beta(\beta-1)-2 \alpha \beta-2 r\right)=0, a_{0} \neq 0
$$

which has the same roots, $\rho_{1}, \rho_{2}$ as those given by (48).

There are two linearly independent solutions to the homogeneous equation (64):

$$
\left.\begin{array}{l}
\phi_{1}(x)=x^{\rho_{1}}\left(1+\sum_{n=1}^{\infty}\left(\frac{\left(-\frac{2 \alpha}{D \sigma^{2}}\right)^{n} \prod_{m=1}^{n}\left(\rho_{1}+m-1\right)}{n ! \prod_{m=1}^{n}\left(m+\frac{\sqrt{\left(2 \alpha+\sigma^{2}\right)^{2}+8 r \sigma^{2}}}{\sigma^{2}}\right)}\right) x^{n}\right) \\
\phi_{2}(x)=x^{\rho_{2}}\left(1+\sum_{n=1}^{\infty}\left(\frac{\left(-\frac{2 \alpha}{D \sigma^{2}}\right)^{n} \prod_{m=1}^{n}\left(\rho_{2}+m-1\right)}{n ! \prod_{m=1}^{n}\left(m-\frac{\sqrt{\left(2 \alpha+\sigma^{2}\right)^{2}+8 r \sigma^{2}}}{\sigma^{2}}\right)}\right) x^{n}\right)
\end{array}\right)
$$

To test the convergence of (65) and (66) we conduct the ratio test. For (65)

$$
\lim _{n \rightarrow \infty}\left|\left(-\frac{2 \alpha x}{D \sigma^{2}}\right) \frac{\left(\rho_{1}+n\right)}{(n+1)\left(n+1+\frac{\sqrt{\left(2 \alpha+\sigma^{2}\right)^{2}+8 r \sigma^{2}}}{\sigma^{2}}\right.}\right| \rightarrow 0
$$

and for (66)

$$
\left.\lim _{n \rightarrow \infty} \mid\left(-\frac{2 \alpha x}{D \sigma^{2}}\right) \frac{\left(\rho_{1}+n\right)}{(n+1)\left(n+1-\frac{\sqrt{\left(2 \alpha+\sigma^{2}\right)^{2}+8 r \sigma^{2}}}{\sigma^{2}}\right.}\right) \mid \rightarrow 0
$$


Both $\phi_{1}(x)$ and $\phi_{2}(x)$ are therefore convergent.

The Wronskian, $W(x)$, of the two linearly independent solutions is given by Abel's formula

$$
W(x)=\exp \left(\int_{x}^{2 \alpha x^{\prime}\left(1-\frac{x^{\prime}}{D}\right)} \frac{\sigma_{2} x^{\prime 2}}{\sigma^{\prime}}\right)=x^{\frac{2 \alpha}{\sigma^{2}}} e^{\frac{2 \alpha}{D \sigma^{2}} x}
$$

which does not vanish anywhere except at $x=0$.

The particular solution to the inhomogeneous equation (63) is then

$$
\Phi(x)=\psi_{1}(x) \phi_{1}(x)+\psi_{2}(x) \phi_{2}(x)
$$

where

$$
\psi_{1}(x)=\int_{x} \frac{\left(p \alpha x^{\prime}\left(1-\frac{x^{\prime}}{D}\right)-h x^{\prime}\right)}{W\left(x^{\prime}\right)} d x^{\prime}, \quad \psi_{2}(x)=\int_{x} \frac{\left(h x^{\prime}-p \alpha x^{\prime}\left(1-\frac{x^{\prime}}{D}\right)\right) \phi_{1}\left(x^{\prime}\right)}{W\left(x^{\prime}\right)} d x^{\prime}
$$

The functions $\psi_{1}(x)$ and $\psi_{2}(x)$ are power series expressions obtained by straightforward term by term integration.

The general solution to the inhomogeneous equation (63) is then

$$
V_{2}^{*}(x)=k_{1} \phi_{1}(x)+k_{2} \phi_{2}(x)+\Phi(x)
$$

where $k_{1}$ and $k_{2}$ are coefficients to be determined by the appropriate boundary conditions. We assume that the function $V_{2}^{*}(x)$ coincides with its counterpart (49) for $D \rightarrow \infty$. It follows from this assumption that $k=0$, hence

$$
V_{2}^{*}(x)=k_{1} \phi_{1}(x)+\Phi(x)
$$

At the threshold point, $x^{*},\left(\frac{d V_{2}^{*}}{d x}\right)_{x=x^{*}}=c$, hence

$$
k_{1}=\frac{c-\Phi^{\prime}\left(x^{*}\right)}{\phi_{1}^{\prime}\left(x^{*}\right)}
$$

Thus, the desired solution to the inhomogeneous equation (63) assumes its final form

$$
V_{2}^{*}(x)=\left(\frac{c-\Phi^{\prime}\left(x^{*}\right)}{\phi_{1}^{\prime}\left(x^{*}\right)}\right) \phi_{1}(x)+\Phi(x)
$$


To ensure continuity of the function at $x^{*}$, we use again the boundary conditions (41):

$$
\begin{aligned}
\left(\frac{d^{2} V_{2}^{*}}{d x^{2}}\right)_{x=x^{*}} & =a_{2} \\
& =\frac{r}{\sigma^{2}\left(x^{*}\right)^{2}} V_{2}^{*}\left(x^{*}\right)+\frac{\alpha(c-p)\left(1-\frac{x^{*}}{D}\right)+h}{\sigma^{2} x^{*}}
\end{aligned}
$$

Also

$$
\begin{aligned}
\left(\frac{d^{2} V_{2}^{*}}{d x^{2}}\right)_{x=x^{*}} & =a_{2} \\
& =\left(\frac{c-\Phi^{\prime}\left(x^{*}\right)}{\phi_{1}^{\prime}\left(x^{*}\right)}\right) \phi_{1}^{\prime \prime}(x)+\Phi^{\prime \prime}(x)
\end{aligned}
$$

Equating (69) and (70) we obtain a single equation in one unknown, $x^{*}$ :

$$
\begin{aligned}
& \frac{c-\Phi^{\prime}\left(x^{*}\right)}{\phi_{1}^{\prime}\left(x^{*}\right)}\left(\phi_{1}^{\prime \prime}(x)-\frac{r}{\sigma^{2}\left(x^{*}\right)^{2}} \phi_{1}\left(x^{*}\right)\right)+\Phi^{\prime \prime}(x)-\frac{r}{\sigma^{2}\left(x^{*}\right)^{2}} \Phi\left(x^{*}\right) \\
& -\frac{\alpha(c-p)\left(1-\frac{x^{*}}{D}\right)+h}{\sigma^{2} x^{*}}=0
\end{aligned}
$$

Solution of (71) through a root-finding scheme should furnish the strategy threshold point, $x^{*}$, with any prescribed accuracy.

\section{Discussion}

In this paper we have solved first the deterministic optimal control problem of discounted net revenue maximisation for inventory driven by demand rate following logistic growth for various regimes of the order rate. We have also obtained the optimum stock level that stabilises demand. We have opted for a logistically driven demand rate motivated by the ubiquity of the logistic equation in other areas of research. Montroll (1978) has argued that production of a product or an industry typically grows drawing the logistic curve. An obvious implication of the logistic growth of an individual product is that if the product enjoys high demand a high order rate for this product is a natural response. Also to keep the control problem autonomous and singular we have used a linear order cost function as opposed to a more traditional polynomial form. We have not considered stock deterioration which might introduce an explicit time dependence in the model, as for instance in the case of perishable goods, and render the control problem non-autonomous but solely stock dependent deterioration can be easily incorporated. Losses from deterioration directly proportional to the present stock, $\theta x$, result in a reduction to the 
optimum switch level in (10) by the amount $\frac{D c \theta}{2 \alpha(p-c)}$. This is reasonable as maintaining a lower stock makes up for the reduction in the net profit due to increased order rates.

The MRAP can be calculated when the holding cost function is of the form, $h(x)=$ $h_{0} x^{\gamma}$, as in Berman and Perry (2006), or inventory deterioration is expressed as a nonlinear function of the inventory, or when the demand rate is a generalised logistic, for instance, $\alpha x^{\beta}\left(1-\frac{x}{D}\right)$, but the resulting Euler-Lagrange is a transcendental equation in $x$ which may possess more than one real root. The optimal path would be the MRAP from the existing inventory level to one of the roots.

Another and perhaps the most interesting research avenue is the problem of pricing. We have used a fixed price, $p$, in our model but demand and price are traditionally interdependent. There are three ways the price variable can enter the model:

1 as an invertible function of demand rate alone, for instance, $p=\lambda F(\dot{y})$

(Gallego and Van Ryzin, 1994), in which case the autonomy of the optimal control problem is maintained

2 as another decision variable with its own state equation evolving in time

3 as a control parameter directly affecting demand as in Jørgensen and Kort (2002).

This paper also solves the stochastic optimal control problem of maximising the net profit from selling an item that is subject to stock-dependent demand and random perturbations due to variations in the inventory and/or demand. The associated H-J-B equation is formulated and solved in two different cases:

1 when the stock level, $D$, that imposes a zero rate on demand growth, is finite

2 when $D$ is infinite.

It is interesting to note that the deterministic version (no random perturbations) of the problem in case $(2)(D \rightarrow \infty)$, does not allow the determination of a switch inventory state, $x^{*}$. As the control variable appears linearly within the equation, the optimal strategy is to employ the extreme values of control to construct the optimal strategy. One strategy is to allow the inventory to drop (no orders) if it happens to be above a threshold level and the other strategy is to build the inventory up to the threshold as fast as possible (maximum possible order) when it falls below the threshold. Once the threshold level, where the marginal profit equals the order cost per unit item, is attained the order rate should be such that the level is maintained on average. An explicit analytical expression for the threshold was only possible for infinite $D$ (case 2). For finite D (case 1) the H-J-B equations assume power series solutions that can deliver the threshold value by appealing to numerical methods. We have found that the threshold level increases with a decrease in uncertainty present in the inventory dynamics, which reasonably suggests that it is more profitable to let the inventory drop to low levels when random fluctuations are persistently large. 
Finally, it is worth pointing out that for very small noise values, $\sigma$, Fleming and Souganidis (1986) have proposed a power series for the form of the value function, $V(x)=\sum_{n=0}^{\infty} \sigma_{n} v_{n}(x)$. Substitution of this series into the H-J-B equation and collecting terms with the same orders of $\sigma$ yields a series of differential equations, with $v_{0}(x)$ being the current value function for the unperturbed problem.

Subsequent equations are first-order linear differential equations in $v_{n}$ with coefficients depending on $v_{n-1}$. The equations for $v_{n}$ can be then solved recursively.

The novel contribution of this work is the determination of an optimal inventory level modified by a stock-dependent demand rate of logistic nature, evolving either deterministically or stochastically. This is important in practice for planning inventory levels that maximise net profit, in the presence of possible uncertainty in demand. However, there are some key limitations in the existing model. The most important limitation is the assumption of a constant price. We focus on introducing price dynamics as an additional factor directly affecting demand in our future research.

\section{References}

Alfares, H.K. (2007) 'Inventory model with stock-dependent demand rate and variable holding cost', International Journal of Production Economics, Vol. 108, No. 1-2, pp.259-265.

Aoki, M. and Yoshikawa, H. (2002) 'Demand saturation-creation and economic growth', Journal of Economic Behavior and Organization, Vol. 48, No. 2, pp.127-154.

Baker, R.C. and Urban, T.L. (1988) 'A deterministic inventory system with an inventory-level-dependent demand rate', The Journal of the Operational Research Society, Vol. 39, No. 9, pp.823-831.

Balakrishnan, A., Pangburn, M.S. and Stavrulaki, E. (2004) 'Stack them high, let'em fly": lotsizing policies when inventories stimulate demand', Management Science, Vol. 50, No. 5, pp.630-644.

Bensoussan, A., Sethi, S.P., Vickson, R. and Derszko, N. (1984) 'Stochastic production planning with production constraints', SIAM Journal of Control and Optimization, Vol. 22, No. 6, pp.920-935.

Berling, P. and Martinez-de-Albéniz, V. (2011) 'Optimal inventory policies when purchase price and demand are stochastic', Operations Research, Vol. 59, No. 1, pp.109-124.

Berman, O. and Perry, D. (2006) 'An EOQ model with state-dependent demand rate', European Journal of Operational Research, Vol. 171, No. 1, pp.255-272.

Chen, Y., Lu, Y. and $\mathrm{Xu}, \mathrm{M}$. (2012) 'Optimal inventory control policy for periodic-review inventory systems with inventory-level-dependent demand', Naval Research Logistics, Vol.59, No. 6, pp.430-440.

Datta, T.K. and Paul, K. (2001) 'An inventory system with stock-dependent, price-sensitive demand rate', Production Planning and Control, Vol. 12, No. 1, pp.13-20.

Dohi, T., Kaio, N. and Osaki, S. (1995) 'Optimal production planning under diffusion demand pattern', Mathematical and Computer Modelling, Vol. 21, No. 11, pp.35-46.

Feichtinger, G. and Hartl, R. (1985) 'Optimal pricing and production in an inventory model', European Journal of Operational Research, Vol. 19, No. 1, pp.45-56.

Fleming, W.H and Rishel, R.W. (1975) Deterministic and Stochastic Optimal Control, Springer Verlag, New York.

Fleming, W.H and Souganidis, P.E. (1986) 'Asymptotic series and the method of vanishing viscosity', Indiana University Mathematics Journal, Vol. 35, No. 2, pp.425-447. 
Fleming, W.H., Sethi, S.P. and Soner, H.M. (1987) 'An optimal stochastic production planning problem with randomly fluctuating demand', SIAM Journal of Control and Optimization, Vol. 25, No. 6, pp.1494-1502.

Gallego, G. and Van Ryzin, G. (1994) 'Optimal dynamic pricing of inventories with stochastic demand over finite horizons', Management Science, Vol. 40, No. 8, pp.999-1020.

Giri, B.C., Pal, S., Goswami, A. and Chaudhuri, K.S. (1996) 'An inventory model for deteriorating items with stock-dependent demand rate', European Journal of Operational Research, Vol. 95, No. 3, pp.604-610.

Goh, M. (1992) 'Some results for inventory models having inventory level dependent demand rate', International Journal of Production Economics, Vol. 27, No. 2, pp.155-160.

Hartl, R. and Feichtinger, G. (1987) 'A new sufficient condition for most rapid approach paths', Journal of Optimization Theory and Applications, Vol. 54, No. 2, pp.403-411.

Jørgensen, S. and Kort, P.M. (2002) 'Optimal pricing and inventory policies: centralized and decentralized decision making', European Journal of Operational Research, Vol. 138, No. 3, pp.578-600.

Kamien, M. and Schwartz, N.L (2001) Dynamic Optimization the Calculus of Variations and Optimal Control in Economics and Management, North Holland.

Khmelnitsky, E. and Gerchak, Y. (2002) 'Optimal control approach to production systems with inventory-level-dependent demand', IEEE Transactions on Automatic Control, Vol. 47, No. 2 , pp.289-292.

Levin, R.I., McLaughin, C.P., Lemone, R.P. and Kottas, J.F. (1972) Production/Operations Management: Contemporary Policy for Managing Operating Systems, McGraw-Hill, New York.

Malliaris, A.G. and Brock, W.A. (1982) Stochastic Methods in Economics and Finance, North Holland.

Montroll, E.W. (1978) 'Social dynamics and the quantifying of social forces', Proceedings of National Academy of Sciences, USA, Vol. 75, No. 10, pp.4633-4637.

Nahmias, S. (2000) Production and Operation Analysis, McGraw-Hill/Irwin, New York.

Sarkar, B., Sana, S.S. and Chaudhuri, K. (2011) 'An economic production quantity model with stochastic demand in an imperfect production system', International Journal of Services and Operations Management, Vol. 9, No. 3, pp.259-283.

Sethi, S.P. and Thompson, G.L. (1981) Applied Optimal Control, Nijhoff, Boston.

Sethi, S.P., Soner, H.M., Zhang, Q. and Jiang, J. (1992) 'Turnpike sets and their analysis in stochastic production planning problems', Mathematics of Operations Research, Vol. 17, No. 4, pp.932-950.

Spence, M. and Starrett, D. (1975) 'Most rapid approach paths in accumulation problems', International Economic Review, Vol. 16, No. 2, pp.388-403.

Tsoularis, A. and Wallace, J. (2002) 'Analysis of logistic growth models', Mathematical Biosciences, Vol. 179, No. 1, pp.21-55.

Urban, T.L. (1992) 'An inventory model with an inventory-level-dependent demand rate and relaxed terminal conditions', Journal of the Operational Research Society, Vol. 43, No. 7 , pp.721-724.

Urban, T.L. (1995) 'Inventory models with the demand rate dependent on stock and shortage levels', International Journal of Production Economics, Vol. 40, No. 1, pp.21-28.

Urban, T.L. (2005) 'Inventory models with inventory-level-dependent demand: a comprehensive review and unifying theory', European Journal of Operational Research, Vol. 162, No. 3, pp.792-804.

Wolfe, H.B. (1968) 'A model for control of style merchandise', Industrial Management Review, Vol. 9, No. 2, pp.69-82. 
Xu, H., Yao, D.D. and Zheng, S. (2011) 'Optimal control of replenishment and substitution in an inventory system with nonstationary batch demand', Productions and Operations Management, Vol. 20, No. 5, pp.727-736.

$\mathrm{Xu}, \mathrm{N}$. (2009) 'Optimal policy for a dynamic, non-stationary, stochastic inventory problem with capacity commitment', European Journal of Operational Research, Vol. 199, No. 2, pp.400-408 